\title{
CASO 5-2013: Femenina 39 años con Heterotopia Pancreática
}

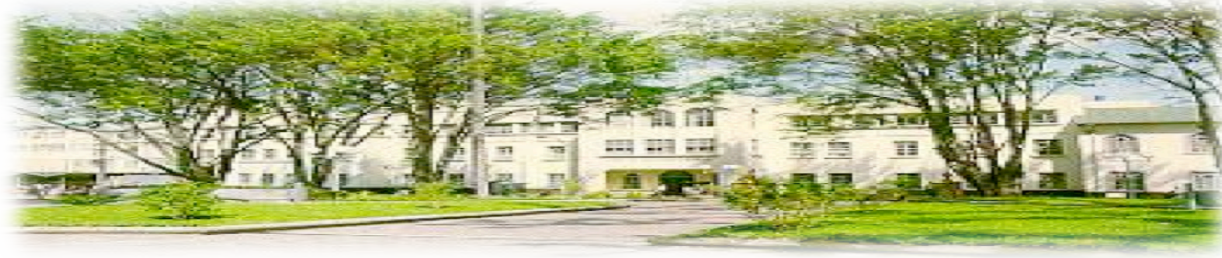

Hospital San quan de Dias. San Dosé. Costa Rica. Fundado en 1845

\section{Reporte de Caso y Revisión de la Literatura}

$\begin{array}{ll}\text { Recibido: } & 12 / 03 / 2013 \\ \text { Aceptado: } & 24 / 04 / 2013\end{array}$

\author{
Rafael Portuguez Barboza ${ }^{1}$ \\ Daniel Zúñiga Monge ${ }^{2}$ \\ Amalia Barboza Solís ${ }^{3}$ \\ Ruy Vargas Baldares ${ }^{3}$
}

\footnotetext{
${ }^{1}$ Especialista en Anatomía Patológica. Servicio de patología, Hospital Max Peralta de Cartago. Profesor de Histologia. Universidad de Costa Rica. Correo electrónico: rportugu@yahoo.com

${ }^{2}$ Médico Cirujano. Profesor de Anatomía. Universidad de Costa Rica. Correo: dr.danielzuniga@medicos.cr. ${ }^{3}$ Médico Residente de Patología. Programa de estudios de posgrado UCR-CENDEISSS. Servicio de Patología. Hospital Max Peralta de Cartago
}

\section{RESUMEN}

La heterotopia pancreática (HP) es una malformación asintomática, que se puede presentar en cualquier lugar del tracto gastrointestinal. En algunas ocasiones presenta síntomas, los cuales varían dependiendo de la localización de la lesión. El tratamiento quirúrgico se emplea en pacientes sintomáticos o en caso de malignidad. Las lesiones asintomáticas benignas se manejan de forma conservadora.

\section{PALABRAS CLAVE}

Heterotopia pancreática. Ultrasonido endoscópico. Malignidad. Cirugía.

\begin{abstract}
Pancreatic heterotopia (HP) is an asymptomatic malformation that can occur anywhere in the gastrointestinal tract. Occasionally symptoms can occur depending on the location of the lesion. Surgical treatment is recommended in symptomatic patients or if malignancy is present. Benign asymptomatic lesions are managed conservatively.
\end{abstract}

\section{KEY WORDS}

Pancreatic heterotopia. Endoscopic ultrasound. Malignancy. Surgery. 


\section{CASO CLÍNICO}

Paciente femenina de 39 años, sin antecedentes patológicos y no patológicos de importancia. Consultó por epigastralgia de tres años de evolución, que aumentó progresivamente en frecuencia e intensidad, asociada a vómitos ocasionales y saciedad precoz.

Se le realizó dos gastroscopias, la primera en agosto de 2009, que documentó masa submucosa de $15 \mathrm{~mm}$ en cuerpo inferior. Se indicó manejo conservador e inhibidores de bomba de protones por 12 semanas. La segunda se realizó en febrero de 2010; se encontró masa de similar tamaño, sin embargo, la paciente persistía con síntomas, por lo cual se indica ultrasonido endoscópico (EUS), donde se observó lesión subepitelial erosionada en su superficie, a nivel de la curvatura menor del cuerpo inferior. Dicha masa comprendía desde la submucosa hasta la muscular propia. Se ralizó el diagnóstico presuntivo de tumor del estroma gastrointestinal (GIST, por sus siglas en inglés).

La tomografía de abdomen simple y con contraste no documentó compromiso linfático o diseminación a tejidos adyacentes. El ultrasonido de abdomen tampoco encontró alteraciones de importancia.

Estudios de laboratorio: hemograma normal, HBsAg negativo, Anti-HCV negativo, NU 3.2 $\mathrm{mg} / \mathrm{dl}$, Crea $0.58 \mathrm{mg} / \mathrm{dl}$, Calcio $7.91 \mathrm{mg} / \mathrm{dl}, \mathrm{Cl}^{-}$ $111 \mathrm{mmol} / 1$. Grupo $\mathrm{O} \mathrm{Rh}(+)$. HIV negativo. VDRL no reactivo. TTP $35.1 \mathrm{~s}$.

Fue llevada a sala de operaciones, donde fue sometida a resección gástrica en cuña, con plastía de la curvatura menor. Se envió tres biopsias a patología, que fueron reportadas como heterotopia pancreática tipo I (total); con lo cual se confirma este diagnóstico y se descarta un GIST.

La paciente evolucionó con buen estado general y sin complicaciones. Se programó controles semestrales con EUS, los últimos de ellos denotan una buena cicatrización de la herida quirúrgica sin reaparición de la masa submucosa. Figura 1. Extracción de la masa tumoral
submucosa

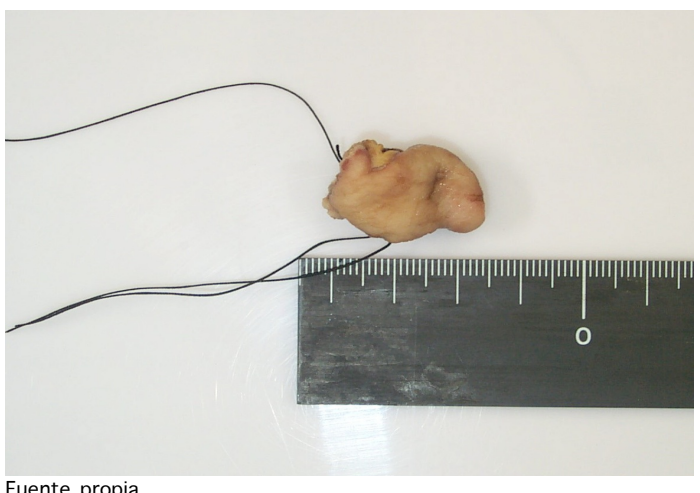

Fuente propia

\section{DISCUSIÓN}

La heterotopia pancreática, también llamada páncreas ectópico o páncreas aberrante ${ }^{(1)}$, se define como la presencia de tejido pancreático que carece de comunicación anatómica o vascular con el órgano pancreático. La frecuencia en estudios de autopsias oscila entre $0.5 \%$ y $13.7 \%{ }^{(2)}$. Es ligeramente más frecuente en hombres de 30 a 50 años ${ }^{(3)}$. Se localiza en un $85 \%$ en el tracto gastrointestinal ${ }^{(4)}$.

Es más comúnmente encontrado en el estómago (a su vez más frecuente en curvatura mayor antral) (25-38\%), duodeno (17-36\%) y yeyuno (15-21\%). También se puede hallar raramente en esófago, vías biliares, vesícula, bazo $\mathrm{y}$ mesenterio $^{(5)}$.

Hay dos teorías que explican la etiología del páncreas ectópico. La teoría de la pérdida o disrupción, que habla del origen embriológico del páncreas a partir de tejido endodérmico del duodeno primitivo. Normalmente se forma un páncreas ventral (cabeza) y otro dorsal (cuerpo y cola). La teoría explica que al rotar el intestino anterior, algunos elementos del páncreas se separan y se forma tejido pancreático maduro a nivel del tracto gastrointestinal. La segunda teoría o de la metaplasia, detalla que la HP se origina de metaplasia pancreática del endodermo que migra a la submucosa durante la embriogénesis $^{(5)}$.

La HP puede dividirse de acuerdo a su histología, en cuatro tipos según la clasificación 
de Heinrich, modificada por Gaspar Fuentes et al. ${ }^{(6,11)}$ :

- Tipo I o total: asociado a tejido pancreático típico (acinos, conductos e islotes).

- Tipo II o canalicular: presenta conductos solamente.

- Tipo III o exocrino: presenta tejido acinar solamente.

- Tipo IV o endocrino: se encuentran solo células de los islotes pancreáticos.

El páncreas ectópico encontrado en este caso, clasificado como tipo I de la clasificación previamente citada, es histológicamente similar al órgano como tal, se identifican las porción exocrina (ductoacinar) y la porción endocrina caracterizado por islotes de Langerhans, sin presencia de cambios neoplásicos (Figuras 2 y 3).

Clínicamente, los síntomas más comunes son dolor abdominal, náuseas, vómitos y sangrado digestivo. Generalmente se presentan en lesiones mayores a $1.5 \mathrm{~cm}$. El dolor asociado a estas lesiones se debe a secreción de enzimas y hormonas resultantes del tejido ectópico que producen irritación e inflamación tisular ${ }^{(7)}$.

El sitio anatómico en el cual se presenta la masa se relaciona con los síntomas. Las lesiones gástricas son las que con mayor frecuencia presentan síntomas, como dolor epigástrico o síntomas obstructivos, consecuencia de una masa prepilórica $^{(8)}$.

En el caso estudiado, la paciente presentaba epigastralgia crónica por tres de las razones previamente mencionadas: el tamaño del tumor (15 mm, figura 1); la masa se encontraba en el cuerpo gástrico y finalmente, el hecho de que se liberen sustancias endocrinas y exocrinas pancreáticas produce inflamación e irritación de la pared gástrica, manifestándose como dolor epigástrico.

La HP también puede manifestarse con lesiones pancreáticas propiamente dichas, como pancreatitis, quistes y pseudoquistes ${ }^{(9)}$.

La transformación maligna del páncreas ectópico es rara, se ha reportado alrededor de 30 casos en la literatura. De éstos, se ha concluido que su mayoría se presentan en el estómago y son más frecuentes los adenocarcinomas ${ }^{(2)}$.
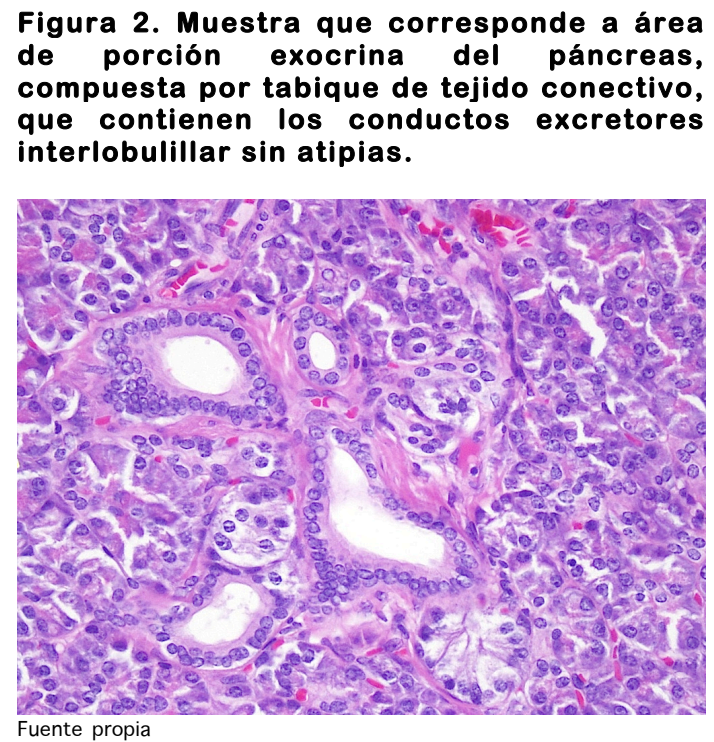

Guillou et al. ${ }^{(10)}$ proponen cuatro criterios para establecer una relación entre la HP y el tejido maligno:

1. La neoplasia debe estar sobre o en las cercanías del tejido ectópico.

2. Debe existir una transición entre el carcinoma y las estructuras pancreáticas normales.

3. Se deben observar ductos y acinos totalmente desarrollados en el tejido heterotópico no neoplásico.

4. Se debe excluir extensión directa o metástasis de otro foco tumoral.

Nuestro caso no cumple con los criterios antes mencionados. Aunque faltan estudios, al parecer el pronóstico es más favorable en pacientes con neoplasias originadas sobre HP que tumores formados sobre el órgano pancreático como tal ${ }^{(4)}$.

Desde el punto de vista radiológico y endoscópico, la HP presenta varias características a saber: en un enema de bario se observa un defecto de llenado, redondeado, con una indentación central, mientras que en la endoscopia se manifiesta como una lesión umbilicada $^{(9)}$. 
Cabe resaltar que estos estudios se realizan con el fin de descartar causas más comunes a algún síntoma relacionado, siendo la HP un hallazgo incidental, tal como ocurrió en nuestro caso, donde la paciente fue estudiada por epigastralgia de larga data y se diagnosticó como GIST antes del resultado de la biopsia.

El EUS es el estandard de oro en el estudio de lesiones submucosas del tracto gastrointestinal. En el antro gástrico, se pueden diagnosticar HP de 0.5 a $2 \mathrm{~cm}^{(11)}$ y combinado con aspirado citológico la sensibilidad es de 80 a $100 \%{ }^{(5)}$.

El manejo de la HP es controversial ${ }^{(12)}$. La resección quirúrgica se recomienda en pacientes sintomáticos, cuya única causa de sus síntomas sea el tejido pancreático ectópico o en formaciones cancerosas. Las lesiones benignas asintomáticas no requieren cirugía. La opinión de expertos está dividida en cuanto a si se debe llevar un control endoscópico o dar el alta médica $^{(5)}$.

En el presente caso, se empleó un manejo quirúrgico debido a los síntomas persistentes de la paciente. Se decidió llevar posteriormente un control endoscópico semestral, con el fin de evaluar la sintomatología o reaparición de alguna lesión de importancia.

Figura 3. Corte histológico que muestra la porción endocrina del páncreas, compuesta por los Islotes de Langerhans sin atipias.

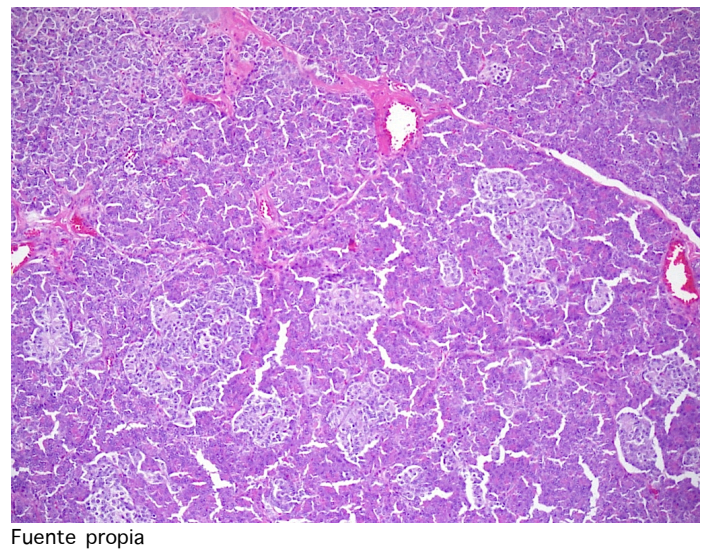

La HP es un hallazgo incidental poco común. Es una lesión generalmente asintomática y benigna; aunque puede manifestarse con síntomas y malignizar. Por lo tanto, es importante tomar al páncreas ectópico dentro del diagnóstico diferencial de una masa submucosa, principalmente a nivel de estómago, duodeno o yeyuno.

En el presente caso, se estableció como primera opción un GIST. La biopsia confirmó la existencia de HP, en una paciente sintomática con una lesión benigna a nivel gástrico en submucosa de cuerpo inferior

\section{BIBLIOGRAFÍA}

1. Inoue $\mathrm{Y}$ Hayashi M Arisaka Y Higuchi K Egashira Y Tanigawa N. Adenocarcinoma arising in heterotopic pancreas (Heinrich type III): a case report. Journal of Medical Case Reports 2010;4:39.

2. Mao-Lin Y Yao-Dong W Yi-Feng T Ying L. Adenocarcinoma arising from intrahepatic heterotopic pancreas: A case report and literature review. World J Gastroenterol 2012; 18(22):2881-2884.

3. Tsapralis D Charalabopoulos A Karamitopoulou E et al. Pancreatic intraductal papillary mucinous neoplasm with concomitant heterotopic pancreatic cystic neoplasia of the stomach: a case report and review of literature. Diagnostic Pathology 2010;5:4.

4. Okamoto H Kawaoi A Ogawara T Fujii H. Invasive Ductal Carcinoma arising from an ectopic pancreas in the gastric wall: a longterm survival case. Case Rep Oncol 2012;5:69-73.

5. Maneesh G. Heterotopic Pancreas. Journal of the Louisiana State Medical Society 2010;162:311-313.

6. Chandan V Wang W. Pancreatic Heterotopia in the Gastric Antrum. Arch Pathol Lab Med 2004;128:111-112.

7. Esquivel C Ballario F García S Giraudo P Granero L. Tumor submucoso gástrico: páncreas heterotópico. Presentación de un caso y revisión de la literatura. Acta Gastroenterol Latinoam 2011;41:234-237.

\section{CONCLUSIONES}


8. Hickman DM Frey CF Carson JW. Adenocarcinoma arising in gastric heterotopic pancreas. West J Med 1981;135:57-62.

9. Sadeghi R Godambe A Shienbaum A Alloy A. Premalignant Gastric Heterotopic Pancreas. Journal of Gastroenterology and Hepatology 2008;4(3):218-221.

10. Guillou L Nordback P Gerber C Schneider R. Ductal adenocarcinoma arising in a heterotopic pancreas situated in a hiatal hernia. Arch Pathol Lab Med 1994;118:568-571.

11. Trifan A Târcoveanu E Mihai D Huţanaşu C Cojocariu C Stanciu C. Gastric Heterotopic Pancreas: an unusual case and review of the literature. J Gastrointestin Liver Dis 2012;21(2):209-212.

12. Yuan Z Chen J Zheng Q Huang XY Yang Z Tang J. Heterotopic pancreas in the gastrointestinal tract. World J Gastroenterol 2009; 15(29):3701-3703. 